\title{
Penning trap assisted decay spectroscopy of neutron-rich ${ }^{115} \mathrm{Ru}$
}

\author{
J. Kurpeta ${ }^{1, a}$, V.-V. Elomaa ${ }^{2}$, T. Eronen ${ }^{2}$, J. Hakala ${ }^{2}$, A. Jokinen ${ }^{2}$, P. Karvonen ${ }^{2}$, I. Moore ${ }^{2}$, H. Penttilä2 \\ A. Płochocki ${ }^{1}$, S. Rahaman ${ }^{2}$, S. Rinta-Antila ${ }^{2}$, J. Rissanen ${ }^{2}$, J. Ronkainen ${ }^{2}$, A. Saastamoinen ${ }^{2}$, T. Sonoda ${ }^{2}$, \\ W. Urban ${ }^{1}$, Ch. Weber ${ }^{2}$, and J. Äystö ${ }^{2}$ \\ 1 Faculty of Physics, Warsaw University, ul.Hoża 69, 00-681 Warsaw, Poland \\ 2 Department of Physics, University of Jyväskylä, P.O. Box. 35, FIN-40351, Jyväskylä, Finland
}

Received: 5 March 2007

Published online: 26 March 2007 - C Società Italiana di Fisica / Springer-Verlag 2007

Communicated by Th. Walcher

\begin{abstract}
Exotic, neutron-rich ${ }^{111} \mathrm{Mo}$ and ${ }^{115} \mathrm{Ru}$ nuclei, produced in proton-induced fission of ${ }^{238} \mathrm{U}$ target, were separated with the IGISOL mass separator. The separator was coupled to the JYFLTRAP Penning trap to select the ions of a single, desired element out of the isobaric IGISOL beam. Monoisotopic samples of ${ }^{115} \mathrm{Ru}$ and ${ }^{111} \mathrm{Mo}$ ions were observed with a microchannel plate detector after the trap or were implanted on a catcher foil for gamma- and beta-ray coincidence spectroscopy. In spite of short data taking time new gamma transitions were identified in the beta decay of very neutron-rich ${ }^{115} \mathrm{Ru}$.
\end{abstract}

PACS. 23.20.Lv $\gamma$ transitions and level energies $-27.60 .+\mathrm{j} 90 \leq A \leq 149$

\section{Introduction}

We report on the beta decay of the exotic, neutron-rich ${ }^{115} \mathrm{Ru}$ isotope, which is located near the neutron and proton midshell. Such nuclei belong to the group of refractory elements and their properties are most conveniently studied with the IGISOL mass separator [1]. For the first time beta and gamma spectroscopy of ${ }^{115} \mathrm{Ru}$ was facilitated by using a Penning trap at the IGISOL, called JYFLTRAP [2]. The beta decay of ${ }^{115} \mathrm{Ru}$ with one associated $\gamma$-ray at $292.8 \mathrm{keV}$ was first reported in [3]. No other spectroscopy information on its decay scheme exists in the literature. The very exotic ${ }^{111} \mathrm{Mo}$ isotope, first identified in the projectile fission of ${ }^{238} \mathrm{U}$ with the fragment separator (FRS) [4], was also observed after the trap. Its yield might be sufficient for a future spectroscopic study employing isobar cleaning with the JYFLTRAP.

\section{Experiment}

The nuclei of interest were produced in the fission of a natural uranium target induced by $25 \mathrm{MeV}$ protons. Fission products were on-line mass separated with the recently upgraded IGISOL 3 [5] mass separator. The ions of a selected mass number $A$ were guided with a 30 degree electrostatic bend to a radio-frequency cooler and buncher [6]. Cooled ions were collected in the cooler and released as a

\footnotetext{
a e-mail: jkurpeta@mimuw.edu.pl
}

short bunch to the JYFLTRAP purification trap. The purification cycle consisting of additional cooling in the trap, a dipole excitation of the ions to a larger radius, a mass selective quadrupole excitation centering the ions that are in resonance with the excitation frequency, and finally the release of the centered ions through a $2 \mathrm{~mm}$ aperture was employed, allowing an isobaric cleaning of the ion bunch. Typical total length of the purification cycle in these experiments was of the order of $120 \mathrm{~ms}$. The purification technique is described in more detail in refs. $[7,8]$. The cleaned ion samples released from the trap were either detected with a microchannel plate (MCP) detector, or implanted into a tape located a few dozens of centimeters behind the MCP. The tape was used to remove the long-lived decay products at regular intervals. The spectroscopy took place at the implantation point. Our spectroscopy setup consisted of two 4-fold segmented super clover detectors of the VEGA array for gamma rays, two low-energy Ge detectors for low-energy gamma rays and a $2 \mathrm{~mm}$ thick plastic scintillator surrounding the collection point for detecting electrons from $\beta$ decay. Data acquisition system was triggered by a signal from any of the clovers or the $\beta$ scintillator or any of the low-energy Ge detectors. Total data taking time for ${ }^{115} \mathrm{Ru}$ was 16 hours.

\section{Results}

The quadrupole electric field of the purification trap was scanned over a wide frequency range covering the whole 


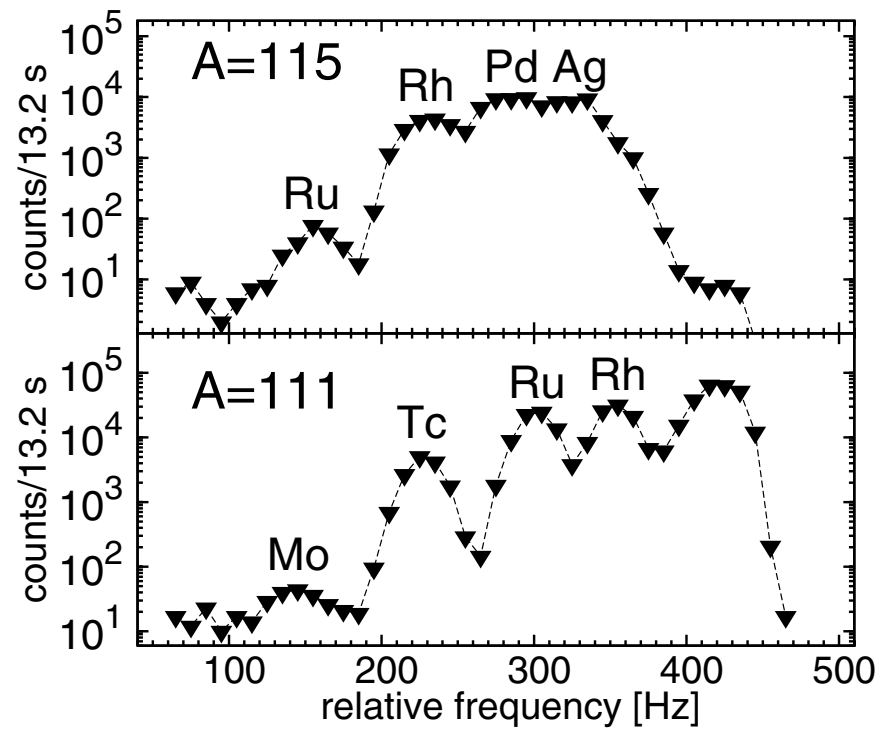

Fig. 1. Ion counts registered with the $\mathrm{MCP}$ detector placed after the purification trap. The well-resolved atomic ions from the IGISOL isobaric beam are marked with the respective element symbol. For the most exotic ${ }^{111}$ Mo there were about 4 ions/s. The total purification cycle time was $121 \mathrm{~ms}$ for $A=$ 115 and $111 \mathrm{~ms}$ for $A=111$.

isobaric chain. The ions leaving the purification trap were registered with the MCP detector. The scans made for $A=111$ and $A=115$ isobars are shown in fig. 1 . The yield of each isotope can be reliably deduced from these scans, as well as the appropriate resonance frequency for their purification. Figure 1 shows how well the isotopes within an isobar can be resolved using the purification trap. The mass-resolving power (MRP) that can be deduced from these scans is of the order of 30000 . In particular, the most neutron-rich nuclei in each isobar are so well separated that the isobaric background for them is totally negligible. The very exotic ${ }^{111}$ Mo nucleus was observed after the trap too, see the lower part of fig. 1 . The yield of ions after the trap was satisfactory for spectroscopic measurements with our setup in the case of ${ }^{115} \mathrm{Ru}$.

In the mass $A=115$ the IGISOL beam was isotopically cleaned to study the decay of a very exotic nucleus ${ }^{115} \mathrm{Ru}$. On the basis of the frequency scan at $A=115$ the purification trap frequency was fixed to correspond to the ${ }^{115} \mathrm{Ru}$ peak frequency. The effect of purification to the isotopic level is presented in fig. 2, which shows two $\gamma$ spectra measured by one of our low-energy Ge detectors. The lower spectrum is measured at the central beam line of the IGISOL facility, from an ion sample, separated with a dipole magnet only. The MRP was about 600 . The upper spectrum is recorded after further purification of the $A=115$ ion beam using the purification trap of the JYFLTRAP. In the spectrum collected after the trap one can clearly see $\gamma$ transitions of ${ }^{115} \mathrm{Ru} \beta^{-}$decay, which are not visible if only the mass separator is used. The nucleus identification is based on a proper quadrupole frequency of the purification trap (found in an earlier scan) and coincidences with the $292.8 \mathrm{keV}$ line known from the previous

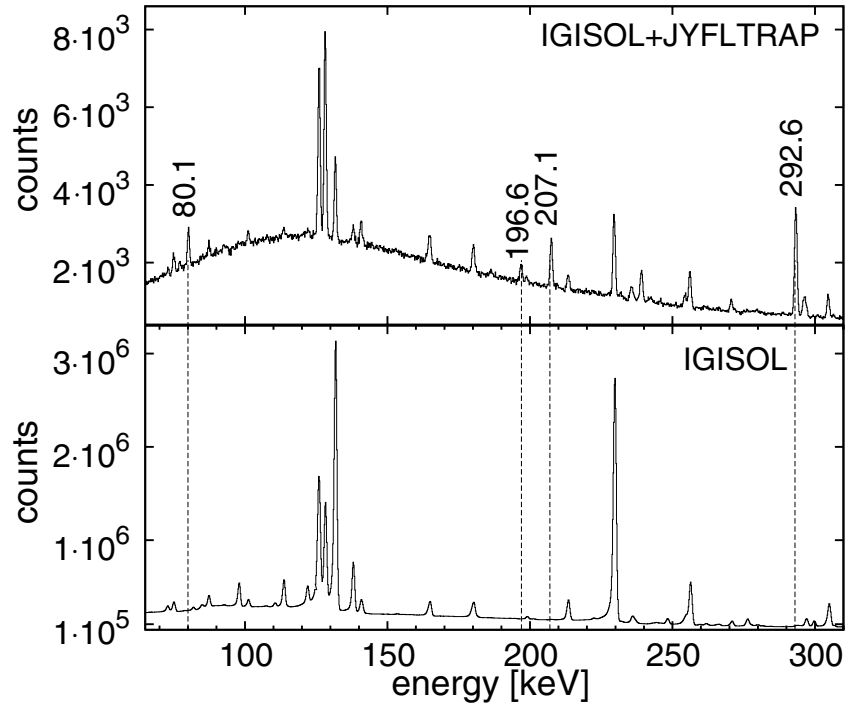

Fig. 2. The upper gamma spectrum was collected with the purification trap set to ${ }^{115} \mathrm{Ru}$. The lower one was recorded at the end of the IGISOL separator, set to mass $A=115$. The peaks ascribed to beta decay of ${ }^{115} \mathrm{Ru}$ are marked with their energies in $\mathrm{keV}$. Vertical lines are to guide the eye.

Table 1. Gamma rays in coincidence with the $292.6 \mathrm{keV}$ line [3], observed after the purification trap, set to ${ }^{115} \mathrm{Ru}$.

\begin{tabular}{rrl}
\hline$E[\mathrm{keV}]$ & $I_{\gamma}^{r e l}[\%]$ & Coincident $\gamma$-lines \\
\hline 80.1 & $12.4(5)$ & 292.6 \\
196.6 & $7.4(4)$ & $207.1,292.6$ \\
207.1 & $25(1)$ & $196.6,292.6$ \\
292.6 & $100(3)$ & $80.1,196.6,207.1$ \\
\hline
\end{tabular}

IGISOL study [3]. Using the coincidence method three new transitions in ${ }^{115} \mathrm{Rh}$ were found: $80.1 \mathrm{keV}, 196.6 \mathrm{keV}$ and $207.1 \mathrm{keV}$. The gated spectra observed in the lowenergy Ge detector are shown in fig. 3 and the resulting coincidence relations are given in table 1 . The observed coincidences and gamma intensities suggest that the three transitions $293 \mathrm{keV}, 207 \mathrm{keV}$ and $197 \mathrm{keV}$ form a cascade in which the $293 \mathrm{keV}$ transition feeds the ground state of ${ }^{115} \mathrm{Rh}$. A partial $\beta$ decay scheme of ${ }^{115} \mathrm{Ru}$, as obtained in this work, is shown in fig. 4 .

In fig. 5 we show a systematics of low-energy excitations in $\mathrm{Ru}$ and $\mathrm{Rh}$ isotopes, which are drown relative to $1 / 2^{+}$and $7 / 2^{+}$levels, respectively. In the $\beta^{-}$decay of $5 / 2^{+}$ground states of ${ }^{109} \mathrm{Ru}$ and ${ }^{111} \mathrm{Ru}$, both groups of medium-spin levels on top of $7 / 2^{+}$ground states and low-spin levels, based on $3 / 2^{+}$excitation are populated in ${ }^{109} \mathrm{Rh}$ and ${ }^{111} \mathrm{Rh}$, respectively. A similar population pattern was observed in the $\beta$ decay of ${ }^{113} \mathrm{Ru}$ to levels in ${ }^{113} \mathrm{Rh}$. This was the reason to propose spin $5 / 2^{+}$also for the ${ }^{113} \mathrm{Ru}$ ground state [9]. However, as we found in our recent study, where both, $\beta$ decay and fission data were analysed, the ground state in ${ }^{113} \mathrm{Ru}$ has spin $1 / 2^{+}[10]$. Beta decay of this state populates low-spin levels in ${ }^{113} \mathrm{Rh}$, while medium-spin levels are populated in the $\beta$ decay of 


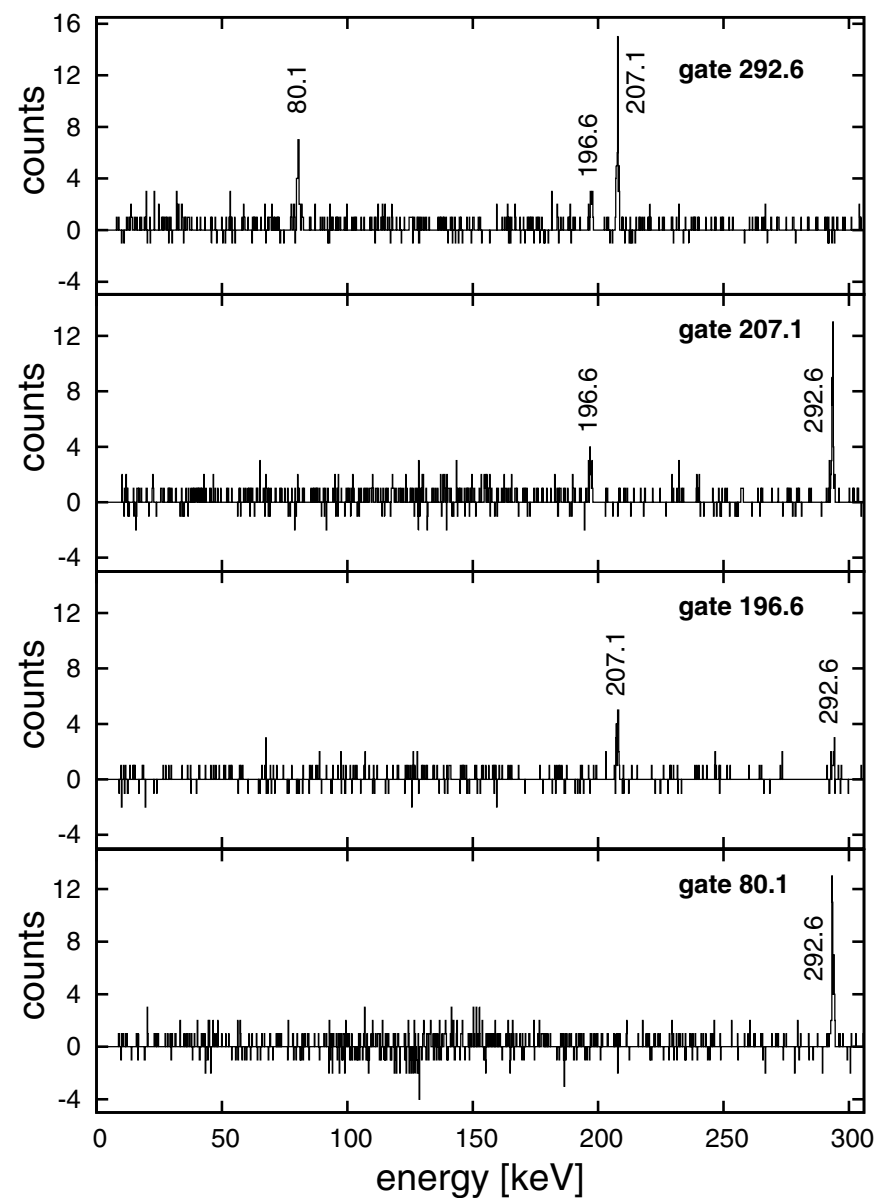

Fig. 3. Coincidence gamma spectra gated on the transitions in ${ }^{115} \mathrm{Ru}$. The gates were set in one of the low-energy Ge detectors and projected to the other one. These spectra were used to construct table 1. Peak energies given in $\mathrm{keV}$.

a $0.5 \mathrm{~s}$ isomeric state in ${ }^{113} \mathrm{Ru}$, which has spin $7 / 2^{-}[10]$. The $5 / 2^{+}$level in ${ }^{113} \mathrm{Ru}$ is located well above the $1 / 2^{+}$ ground state, as seen in the upper part of fig. 5. Thus, the systematics shown in fig. 5 suggest spin $1 / 2^{+}$for the ground state in ${ }^{115} \mathrm{Ru}$. Consequently, $3 / 2^{+}$and $5 / 2^{+}$levels in ${ }^{115} \mathrm{Rh}$ are populated in the $\beta$ decay of ${ }^{115} \mathrm{Ru}$, as evidenced in the lower part of fig. 5 .

There remains an interesting question if the $\beta$-decaying isomer forms only in ${ }^{113} \mathrm{Ru}$ or if it is present also in ${ }^{115} \mathrm{Ru}$. On one hand, the trend shown by the $7 / 2^{-}$level in ${ }^{111} \mathrm{Ru}$ and ${ }^{113} \mathrm{Ru}$ suggest a low-lying $7 / 2^{-}$in ${ }^{115} \mathrm{Ru}$ forming a possible $\beta$-decaying isomer. On the other hand, the lack of the candidate for $9 / 2^{+}$excitation populated in the $\beta$ decay of ${ }^{115} \mathrm{Ru}$ points against such a hypothesis.

The case of the very neutron-rich ${ }^{115} \mathrm{Rh}$ certainly needs further spectroscopic investigations. The presented results show that a combination of the IGISOL mass separator and the JYFLTRAP, which provides isobarically purified ion samples, is a powerful tool for studying the nuclear structure very far from stability.
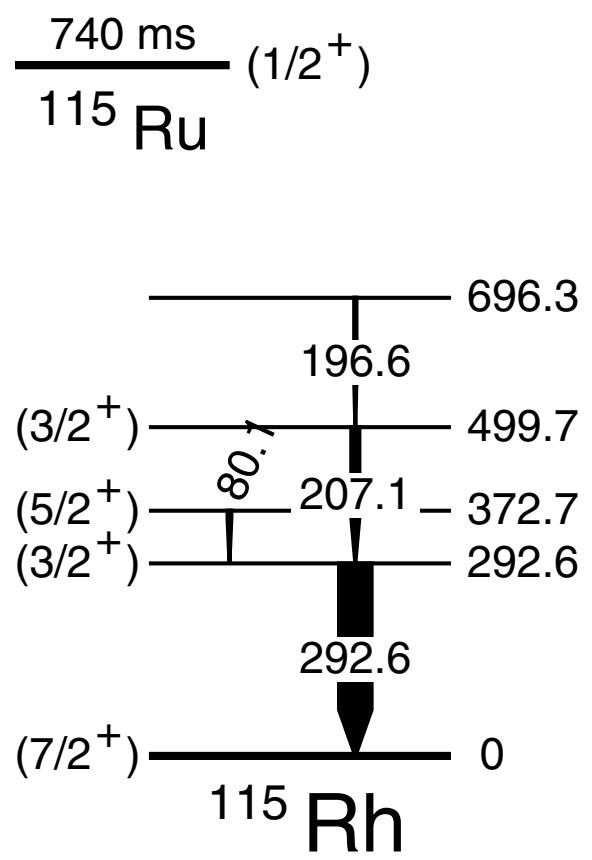

Fig. 4. A partial ${ }^{115} \mathrm{Ru}$ decay scheme as obtained in the present work. $Q_{\beta}$ measured with JYFLTRAP is $8165(11) \mathrm{keV}$ [11] and the half-life was measured earlier at IGISOL [3]. The prediction of the ${ }^{115} \mathrm{Ru}$ ground-state spin is based on [10].

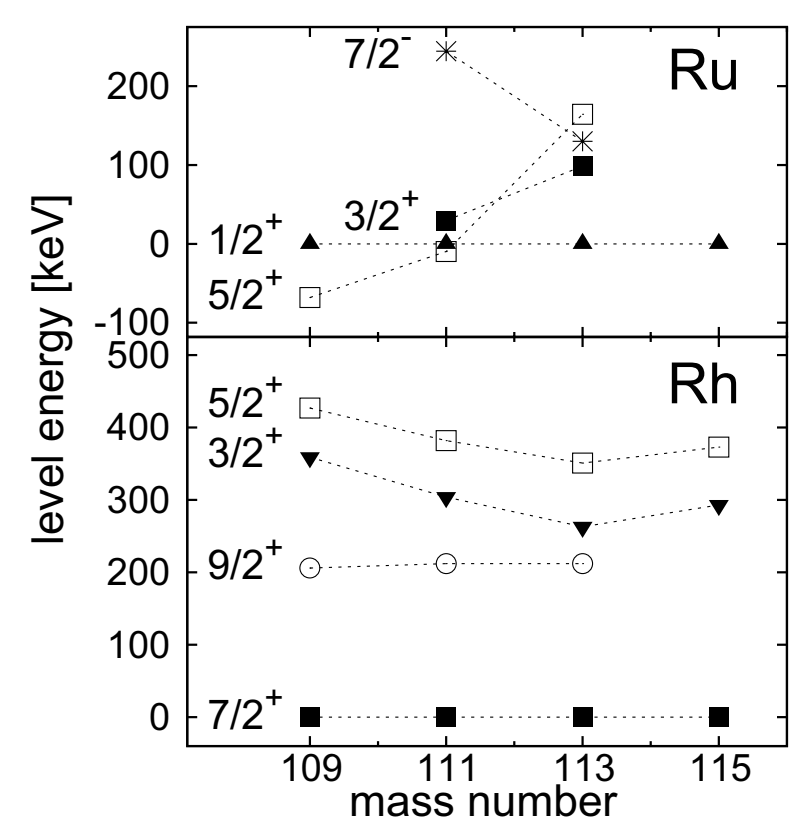

Fig. 5. Low-energy levels in odd $\mathrm{Ru}$ isotopes are shown relative to $1 / 2^{+}$levels. Data for ${ }^{109} \mathrm{Ru}[12],{ }^{111} \mathrm{Ru}[13]$ and the spins of the ground and isomeric states for ${ }^{113} \mathrm{Ru}$ are as proposed in [10]. In the systematics of the low-energy levels in odd-Rh isotopes the data for $A=115$ obtained in this work. 
We would like to thank P. Jones and P. Greenless for help in operating the VEGA cluster detectors. This work has been supported by the EU 6th Framework programme "Integrating Infrastructure Initiative - Transnational Access", Contract Number: 506065 (EURONS) and by the Academy of Finland under project No. 111428 and the Finnish Center of Excellence Programme 2006-2011 (Nuclear and Accelerator Based Physics Programme at JYFL).

\section{References}

1. J. Äystö, Nucl. Phys. A 693, 477 (2001).

2. A. Jokinen et al., Eur. Phys. J. A 25, s1, 27 (2005).
3. J. Äystö et al., Phys. Rev. Lett. 69, 1167 (1992).

4. M. Bernas et al., Phys. Lett. B 331, 19 (1994).

5. H. Penttilä et al., Eur. Phys. J. A 25, s1, 745 (2005).

6. A. Nieminen et al., Nucl. Instrum. Methods A 469, 244 (2001).

7. S. Rinta-Antila et al., Eur. Phys. J. A 31, 1 (2007).

8. U. Hager et al., Phys. Rev. Lett. 96, 042504 (2006).

9. J. Kurpeta et al., Eur. Phys. J. A 13, 449 (2002).

10. J. Kurpeta et al., submitted to Eur. Phys. J. A.

11. U. Hager et al., submitted to Phys. Rev. C.

12. H. Penttilä et al., JYFL Annu. Rep., 77 (1989).

13. W. Urban et al., Eur. Phys. J. A 22, 231 (2004). 